\title{
Comportement antigénique de divers Flagellés au cours des Leishmanioses cliniques et expérimentales
}

\author{
Par J. RANQUE et S. DUNAN
}

L'existence de parentés antigéniques entre les leishmanies et certains micro-organismes biologiquement très éloignés n'est plus à démontrer depuis que l'on a pu effectuer avec succès des réactions de Bordet et Gengou, au cours des leishmanioses cliniques et expérimentales, en se servant d'extraits de bacilles paratuberculeux (1) (2) (3).

Cependant, il s'agit là de propriétés relativement limitées, s'exprimant essentiellement par la fixation du complément.

L'utilisation d'antigènes préparés à partir de divers Flagellés permet une étude plus large comprenant diverses réactions sérologiques et des tests d'allergie cutanée.

Ces Flagellés sont soit des formes Leptomonas des diverses Leishmanies (4) (5), soit des Trypanosomes (6) (7) (8), soit des espèces zoologiquement très différentes telles que les Flagellés d'Euphorbiacées (8) (9) (10) (11).

Pour notre part, depuis six ans, nous avons étudié le comportement antigénique des espèces suivantes :

Trypanosoma equiperdum (T.E.), Leptomonas ctenocephali (L.C.) ; Strigomonas fasciculata (S.F.) et Strigomonas oncopelti (S.O.) (*).

\section{$1^{\circ}$ PREPARATION DES ANTIGENES.}

A) L'ANTIGène A $T$. equiperdum: Technique mise au point avec M. R. Dépieds (6).

La souche de $T$. equiperdum est entretenue par passage intrapéritonéal chez le cobaye. 72 heures après inoculation, l'infestation étant à son maximum, le sang est recueilli par ponction intracardiaque. Mélangé à une solution de citrate à $5 \%$, il est ensuite centrifugé à 2.000 tours/mn durant cinq minutes. Les Trypanosomes se déposent au-dessus des hématies, formant une couche blanchâtre. Après vérification de l'absence de parasites, le surnageant est

* Nous remercions le Docteur Colas-Belcour de l'Institut Pasteur de Paris qui nous a très obligeamment procuré ces souches. 
éliminé à la pipette. La couche de Trypanosomes, soigneusement décollée de celle des hématies, est reprise et mise dans des tubes à hémolyse avec du sérum physiologique.

Une nouvelle centrifugation à grande vitesse permet d'éliminer les hématies restantes. Les Trypanosomes sont récupérés à l'aide d'eau physiologique qui, par aspirations, refoulements doux provoque des courants liquidiens mobilisant les particules de la couche blanche, sans toucher aux hématies.

Ce lavage, répété trois fois, permet d'obtenir un antigène pur, ainsi que le montre la vérification microscopique.

Le dernier culot obtenu est dissous en eau physiologique, l'opacité du liquide trahissant sa richesse, et formolé à $1 \%$. La conservation s'effectue à $-10^{\circ}$.

\section{B) Les ANTIGÈnes A Leptomonas (L.C., S.F. et S.O.).}

Trois souches ont servi à les préparer: deux issues de Leptomonas du puce de chien : Leptomonas ctenocephali (L.C.) et Strigomonas fasciculata (S.F.), une isolée à partir d'Euphorbes : Strigomonas oncopelti (S.O.).

Les trois souches sont cultivées sur milieu liquide composé de bouillon de viande, additionné de sang de lapin défibriné à raison de VIII gouttes par tube de $10 \mathrm{cc}$. Conservées à l'étuve à $26^{\circ} \mathrm{C}$, deux d'entre elles (S.F. et L.C.) se développent en surface, formant un voile blanchâtre adhérant aux parois du tube, tandis que S.O. pousse sur toute la hauteur du tube, donnant ainsi un rendement très supérieur.

Les Flagellés sont à leur maximum de développement au bout de 12 à 15 jours. C'est à ce moment-là que s'effectue la récupération de l'antigène. Pour celle-ci, il suffit de dissocier le voile doucement, à l'aide d'une pipette Pasteur puis de prélever la couche supérieure du tube, sans aspirer d'hématies.

Le liquide ainsi obtenu est centrifugé 20 minutes à 9.000 tours. Il se divise en deux couches :

- le surnageant, constitué par le bouillon de culture dans lequel il ne reste presque plus de parasites, comme le montre l'examen entre lame et lamelle ;

- le culot, formé de Flagellés souvent encore mobiles. Il est, après rejet du surnageant, additionné de sérum physiologique pour être lavé, puis centrifugé à nouveau.

Après cinq lavages, le dernier culot obtenu est constitué d'un amas de flagellés pur. L'antigène sera formé par ce culot, auquel on ajoute une quantité d'eau physiologique merthiolatée à $0,5 \%$, suffisante pour obtenir le degré d'opacité voulu correspondant à 500.000 parasites par $\mathrm{mm}^{3}$. Cet antigène peut être conservé en glacière pendant plusieurs mois.

\section{$2^{\circ}$ UTILISATION DES ANTIGENES.}

Nous avons utilisé essentiellement ces antigènes soit en intradermo-réaction, soit en fixation du complément (réaction de Calmette et Massol et réaction de Kolmer).

Accessoirement, nous avons pratiqué des recherches d'immobilisation spécifique, de précipitation en plaque de gélose (méthodes de Oudin-Ouchterlony) et d'électroimmunophorèse.

Nous avons fait également quelques tentatives d'immuno-fluorescence.

Cependant, les résultats fournis par ces quatre dernières méthodes sont trop frag- 
mentaires et surtout beaucoup trop contradictoires pour pouvoir être pris en considération.

Des études se poursuivent actuellement pour compléter ces premières observations.

\section{A) Les intradermo-réactions}

a) Technique d'utilisation.

L'injection intradermique d'antigène faite au niveau de la région sous-deltoïdienne doit former une papule de $5 \mathrm{~mm}$ de diamètre. Une injection d'eau physiologique formolée à $1 \%$, ou merthiolatée à $0,5 \%$ (selon l'antigène utilisé), pratiquée à côté de la première, servira de témoin.

La réaction est considérée comme positive lorsqu'on obtient une maculo-papule dépassant $1 \mathrm{~cm}$ de diamètre et persistant après 48 heures.

b) Les RÉsultats obtenus.

1) Avec T. equiperdum : la valeur de l'antigène est d'abord confirmée par l'essai du test chez 63 sujets indemnes de parasitose, chez qui la réponse a toujours été négative. Par la suite, il est essayé sur deux séries de sujets : une série de six malades, dont quatre ayant des lésions caractéristiques du bouton d'Orient, avec présence de Leishmania chez deux d'entre eux. Tous quatre présentent un test fortement positif.

Chez les deux autres, les lésions cutanées observées semblent peu spécifiques et l'intradermo reste négative.

Une série de neuf malades atteints de leishmaniose viscérale (à $L$. infantum) avec mise en évidence du parasite dans la moelle osseuse. Chez tous, la réponse a été négative.

Il semble donc qu'il y ait une sensibilisation cutanée à $T$. equiperdum dans le bouton d'Orient, qui n'existe pas dans le kala-azar. Ce fait s'est vérifié aussi chez l'animal.

Deux chiens naturellement infestés par $L$. donovani infantum avec présence de parasites dans les ganglions donnent une réponse négative au test pratiqué sur la face externe de la cuisse, préalablement rasée.

- Une série de 22 hamsters est inoculée : 18 avec une souche de $L$. donovani d'origine canine, 3 avec une souche humaine du même parasite et un avec L. tropica, qui donne chez lui une atteinte viscérale très longue à se développer. Dans tous les cas, l'intradermo-réaction est restée négative.

Il n'y a donc pas de sensibilisation cutanée à $T$. equiperdum chez l'animal inoculé avec $L$. donovani infantum. Dans ce dernier cas, il convient de faire une réserve, car la différence de structure entre la peau humaine et la peau des hamsters peut expliquer ces divergences dans les réponses à une intradermo-réaction.

2) Avec Strigomonas oncopelti: ici encore, aucune réaction chez des sujets non parasités. Par contre, utilisé en Iran par l'un de nous (1) dans le village de Dolat

(1) J. Ranque. 
Abad, près d'Ispahan, chez des malades atteints de bouton d'Orient, il a donné les résultats indiqués dans le tableau ci-après.

Tableau I. - Tests intradermiques (Village de Doalat Abad.)

\begin{tabular}{|c|c|c|c|}
\hline $\mathrm{N}^{\circ:}$ & Noms & Ages & Réactions \\
\hline 1 & Abass Ali Aba... ............ & 4 ans & +++ \\
\hline 2 & Mougtaba Moh... ........... & 2 ans & + \\
\hline 3 & Mohamad Ali Dou... ......... & 6 mois & + \\
\hline 4 & Fatomih Moh... $\ldots \ldots \ldots \ldots$ & 5 ans & ++ \\
\hline 5 & Fatomih Abe... . . . . . . . & 35 ans & + \\
\hline 6 & Mortaza Too... ........... & 8 mois & + \\
\hline 7 & Zohra Kam... ............ & 8 mois & +++ \\
\hline 8 & Ramazan Ali Alz... ......... & 1 an & +++ \\
\hline 9 & Batool Dar... $\ldots \ldots \ldots \ldots \ldots$ & 16 mois & ++ \\
\hline 10 & Ali $\operatorname{Kam} \ldots \quad \ldots \ldots \ldots \ldots \ldots$ & 2 ans & + \\
\hline 11 & Hassan Kam... ............. & 50 ans & 一 \\
\hline 12 & Mougtaba Dou... ........... & 16 mois & \pm \\
\hline 13 & Smat Abe... .............. & 1 an & +++ \\
\hline 14 & Azadiga Esm... $\ldots \ldots \ldots \ldots \ldots$ & 2 ans & + \\
\hline 15 & Nassant Dou... ............ & 10 mois & + \\
\hline 16 & Mohamad Ali Dou... ......... & 6 mois & - \\
\hline 17 & Mougtaba Dou... ............ & 17 mois & \pm \\
\hline
\end{tabular}

Deux réponses négatives ont donc été enregistrées. L'une d'elles s'étant produite chez un enfant, présentant une athrepsie du nouveau-né, ne possède que peu de valeur.

Essayé dans quelques cas de kala-azar, cet antigène n'a donné de réponse positive que deux fois, chez deux malades en provenance du Tchad, dont nous parlerons ultérieurement.

3) Avec Strigomonas fasciculata et Leptomonas ctenocephali : ces deux antigènes n'ont été utilisés en intradermo que dans sept cas, chez des malades hospitalisés à Michel-Lévy dans le Service du Colonel Charmot, dont six présentaient une atteinte à $L$. tropica, vérifiée microscopiquement, et le dernier une trypanosomiase africaine au deuxième stade. Voici les résultats obtenus :

Les circonstances ne nous ont pas permis d'utiliser simultanément les quatre antigènes précités en intradermo-réaction. Il est donc difficile de porter un jugement sur la valeur comparée de ce matériel.

Il semble cependant que (T.E.) et (S.O.) donnent les résultats les plus sensibles tout en étant spécifiques, tandis que S.F. provoque des réactions plus discrètes et L.C. des réponses encore plus faibles.

Nous verrons que cette impression est pleinement confirmée par une expérimentation très valable portant sur la réaction de fixation du complément. 
TABleaU II

\begin{tabular}{|c|c|c|c|c|}
\hline & L.C. & S.F. & s.o. & Trypanosome \\
\hline $\begin{array}{c}1 \\
2 \\
3 \\
4 \\
5 \\
6 \\
7 \\
\text { (Trypanoso- } \\
\text { miase africaine) }\end{array}$ & $\begin{array}{l} \pm \\
+ \\
+ \\
+ \\
+ \\
\pm \\
-\end{array}$ & $\begin{array}{l}+++ \\
+++ \\
+++ \\
+++ \\
+++ \\
+++ \\
-\end{array}$ & $\begin{array}{c}+++ \\
++++\end{array}$ & \pm \\
\hline
\end{tabular}

\section{B) Les fixations du complément}

La présence d'une sensibilité cutanée aux antigènes S.O. et $T$. equiperdum, chez les sujets parasités par L. tropica, laisse supposer l'existence d'une parenté immunologique entre ces différentes espèces.

Cette sensibilité étant absente chez les malades atteints de kala-azar, nous avons été amenés à rechercher chez ceux-ci la présence d'un anticorps sérique pouvant réagir avec les antigènes cités plus haut.

Nous avons pour cela employé la réaction de fixation du complément chez 5 malades porteurs de leishmaniose viscérale, chez 11 chiens naturellement leishmaniés et chez 45 hamsters inoculés à cet effet.

a) LE MATÉRIEL UTILISÉ.

- Le sérum prélevé stérilement, par ponction veineuse chez l'homme et chez le chien et par ponction cardiaque chez le hamster, est décomplémenté par chauffage à $56^{\circ}$ juste avant utilisation.

- Les antigènes employés sont les antigènes S.O., S.F. et L.C. décrits plus haut.

- Le complément, préparé au laboratoire, est constitué par du sérum de cobaye mâle conservé à $-25^{\circ}$. Il doit être titré avant chaque réaction.

- Le système hémolytique comprend des globules rouges de mouton à $5 \%$ et du sérum hémolytique titrant $1,2 \%$.

\section{b) LES TECHNIQUES.}

Les techniques employées sont celles de Calmette et celles de Kolmer, cette dernière présentant l'avantage de ne nécessiter que des quantités faibles de sérum, avantage appréciable quand on s'adresse au sérum de hamster.

Nous avons dû cependant apporter quelques modifications à ces techniques: le pouvoir anticomplémentaire élevé de certains sérums leishmaniés, en particulier des 


\section{Tableau III. - Cas humains}

\begin{tabular}{|c|c|c|c|}
\hline & Clinique & Laboratoire & Traitement \\
\hline 2 & $\begin{array}{l}\text { Age : } 23 \text { ans. } \\
\text { Venant de Kabylie. } \\
\text { Symptômes: } \\
\text { - Hépatosplénumégalie, } \\
\text { - Fièvre, } \\
\text { - Pas de ganglions, } \\
\text { - Association avec le palu- } \\
\text { disme. }\end{array}$ & 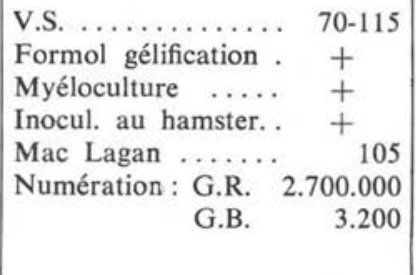 & $\begin{array}{l}\text { Lomidine : } \\
\text { Sujet guéri depuis plus d' } \\
\text { au moment du prélèvem }\end{array}$ \\
\hline 13 & $\begin{array}{l}\text { Age : } 3 \text { ans. } \\
\text { Venant de Bastia. } \\
\text { Symptômes: } \\
\text { - Fièvre, } \\
\text { - Toux, } \\
\text { - Hépatosplénomégalie, } \\
\text { - Ganglions. } \\
\text { Il s'agit d'une rechute. }\end{array}$ & $\begin{array}{lc}\text { V.S. } \ldots \ldots \ldots \ldots . & 49-83 \\
\text { Formol gélification } & + \\
\text { Ponction ganglion } & + \\
\text { Numération : G.R. } & 3.880 .000 \\
\text { G.B. } & 5.200 \\
& \end{array}$ & $\begin{array}{l}\text { Lomidine: } \\
\text { Traité depuis deux jours } \\
\text { a déjà reçu un traitemeı } \\
\text { d'une première atteinte. }\end{array}$ \\
\hline 4 & $\begin{array}{l}\text { Age : } 21 \text { mois. } \\
\text { Venant d'Allauch. } \\
\text { Symptômes: } \\
\text { - Vomissements, } \\
\text { - Diarrhée, } \\
\text { - Amaigrissement, } \\
\text { - Splénomégalie. }\end{array}$ & $\begin{array}{rrr}\text { Formol gélification } & + \text { en } 15 \\
\text { Mac Lagan } \ldots \ldots \ldots & 165 \\
\text { Hanger } \ldots \ldots \ldots & +++ \\
\text { Numération: G.R. } & 3.900 .000 \\
\text { G.B. } & 10.800\end{array}$ & $\begin{array}{l}\text { Glucanthime : } \\
\text { Traité depuis cinq jouri }\end{array}$ \\
\hline 5 & $\begin{array}{l}\text { Age : } 15 \text { mois. } \\
\text { Venant d'Allauch. } \\
\text { Symptômes: } \\
\text { - Troubles digestifs, } \\
\text { - Fièvre anarchique, } \\
\text { - Splénomégalie, } \\
\text { - Hépatomégalie. } \\
\text { - Adénopathies inguinal. }\end{array}$ & $\begin{array}{rr}\text { Formol gélification: } & \text { opacité } \\
\text { légère après } 2 \mathrm{~h} \text { d'étuve. } \\
\text { Ponction médullaire } & + \\
\text { Numération : G.R. } & 3.300 .000 \\
\text { G.B. } & 6.300\end{array}$ & $\begin{array}{l}\text { Glucanthime: } \\
\text { Fin de traitement. }\end{array}$ \\
\hline 7 & $\begin{array}{l}\text { Age : } 30 \text { ans. } \\
\text { Venant de l'Ennedi. } \\
\text { Symptômes: } \\
\text { - Fièvre, } \\
\text { - Splénomégalie, } \\
\text { - Amaigrissement, } \\
\text { - Edèmes, } \\
\text { - Météorisme abdominal. } \\
\text { - Ganglions inguinaux. }\end{array}$ & 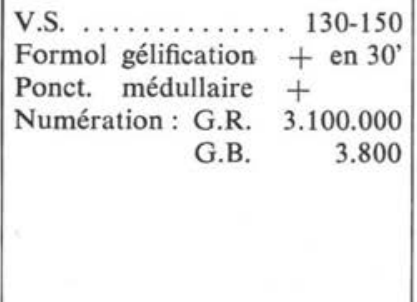 & $\begin{array}{l}\text { Glucanthime et lomid } \\
\text { Fin de traitement. }\end{array}$ \\
\hline 8 & $\begin{array}{l}\text { Age : } 26 \text { ans. } \\
\text { Venant d'Abéché. } \\
\text { Kala-Azar. }\end{array}$ & $\begin{array}{l}\text { Formol gel. } \ldots \ldots \ldots+30^{\prime} \\
\text { Mac Lagan } \ldots \ldots 102 \text { U.V. } \\
\text { Ponct. médul. } \ldots \ldots+\end{array}$ & \\
\hline
\end{tabular}




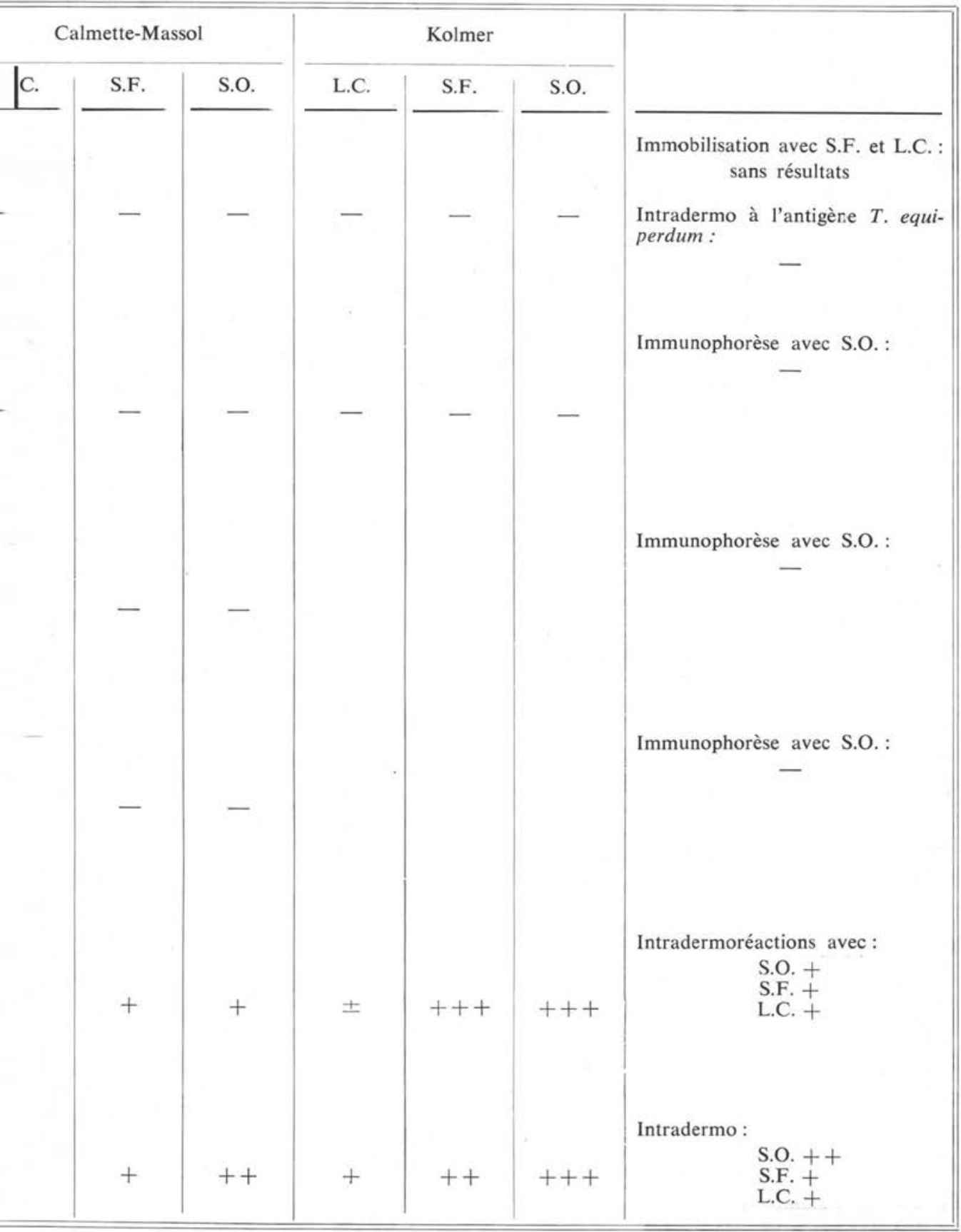




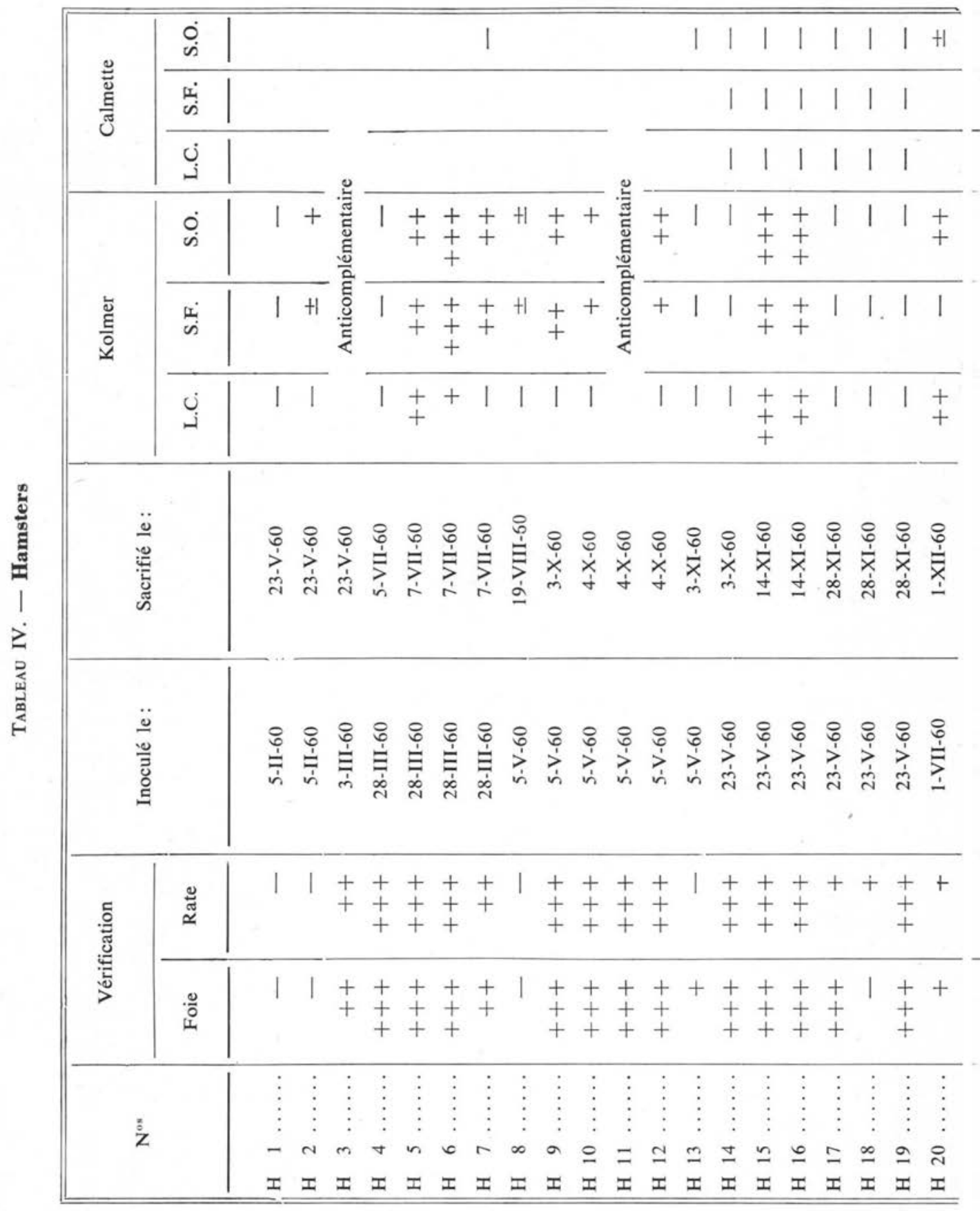




\begin{tabular}{|c|c|c|c|c|c|c|c|c|c|c|c|c|c|c|c|c|c|c|c|c|}
\hline | & & & 1 & 1 & & 1 & I & & & I & & & & 1 & 1 & 1 & & & 1 & ++ \\
\hline & & & 1 & 1 & & 1 & 1 & & & 1 & & \multirow{5}{*}{ 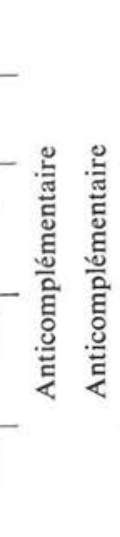 } & & 1 & 1 & 1 & & 1 & 1 & +1 \\
\hline 1 & & & 1 & 1 & & I & I & & & 1 & & & & 1 & 1 & & & + & 1 & $1+$ \\
\hline & $\begin{array}{l}++ \\
++ \\
++\end{array}$ & 胥 & + & $\begin{array}{l}+ \\
+ \\
+\end{array}$ & + & + & + & & & & $\begin{array}{l}+1 \\
+ \\
+\end{array}$ & & + & 1 & + & 1 & + & 늟 & & ++ \\
\hline $\mid$ & $\begin{array}{l}+ \\
+ \\
+\end{array}$ & 苞 & + & + & + & 1 & H & +1 & I & + & +1 & & I & 1 & 1 & 1 & & हूँّ & 1 & $1+$ \\
\hline | & $\begin{array}{l}+ \\
+\end{array}$ & & + & + & + & I & I & 1 & I & 1 & 11 & & H & 1 & 1 & 1 & & 苋 & 1 & $+\begin{array}{r}+ \\
+ \\
+\end{array}$ \\
\hline & 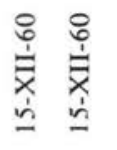 & 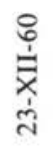 & $\begin{array}{l}\stackrel{P}{0} \\
\dot{\vec{x}} \\
\dot{\vec{\lambda}}\end{array}$ & $\frac{\overline{1}}{\dot{m}}$ & $\frac{\bar{\phi}}{\dot{m}}$ & $\frac{\bar{b}}{\frac{1}{b}}$ & $\frac{\overline{0}}{\frac{1}{\alpha}}$ & $\frac{\overline{0}}{\alpha}$ & 寅 & 京 & 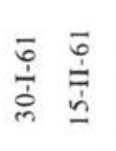 & 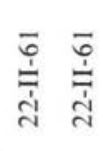 & $\stackrel{\bar{b}}{\stackrel{\Xi}{\Xi}}$ & 京 & 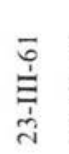 & 㝘 & $\begin{array}{l}\stackrel{\vec{\jmath}}{\Xi} \\
\stackrel{\Xi}{=}\end{array}$ & 站 & $\begin{array}{l}\overrightarrow{0} \\
\stackrel{\Xi}{\Xi}\end{array}$ & 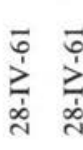 \\
\hline
\end{tabular}

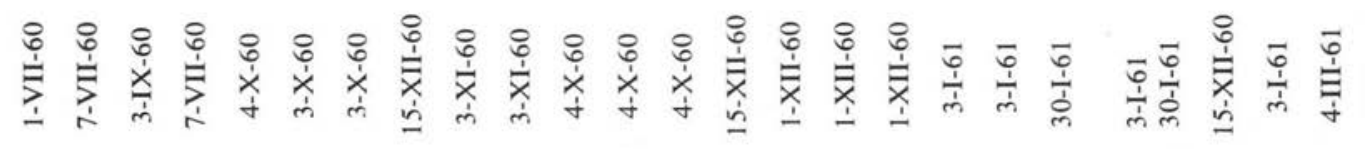

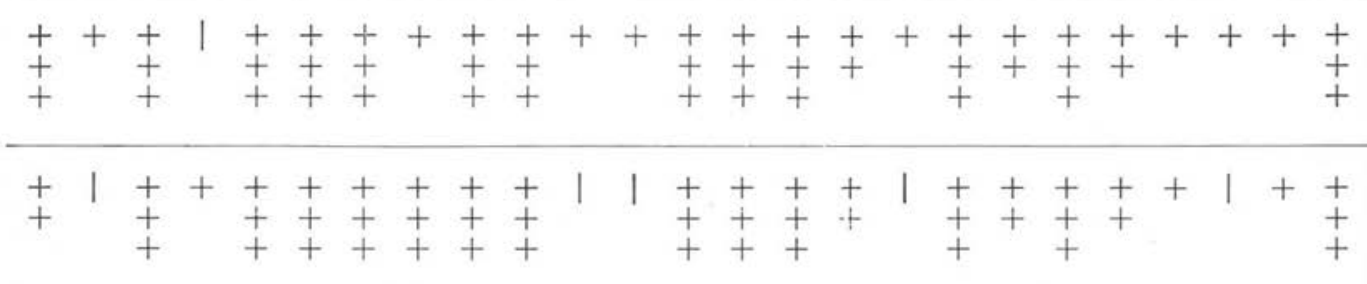

$\overline{4}$ สิ

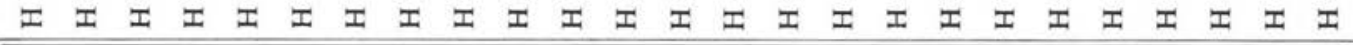


sérums de chiens, conduit à l'utilisation au départ de dilutions faibles de complément (1/10 ou 1/5). Pour la même raison, le titrage du complément doit être fait en présence du sérum. Les antigènes préalablement titrés ne se sont jamais montrés anticomplémentaires.

Pour le reste, nous avons suivi la méthode habituelle :

- Pour la réaction de Kolmer :

- titrage du complément dilué au $1 / 10$, en présence d'une goutte de sérum ;

- utilisation, pour la réaction, de deux unités de complément ;

- fixation pendant 16 heures à la glacière, et, le système hémolytique ayant été ajouté et les tubes réchauffés à l'étuve à $37^{\circ}$ :

- lecture 10 minutes après hémolyse des témoins.

- Pour l'autre réaction, on part du complément dilué au 1/5. Son titrage est effectué par adjonction de doses croissantes à des quantités fixes de sérum, en présence du système hémolytique. Etant donné que le complément a été titré en présence de sérum, on utilise pour la réaction la dose minima active déterminée par ce titrage. La lecture se fait après 30 minutes d'étuve à $37^{\circ}$.

c) Les RÉsultats.

Ils ont été consignés dans les tableaux ci-après :

Tableau V. - Chiens

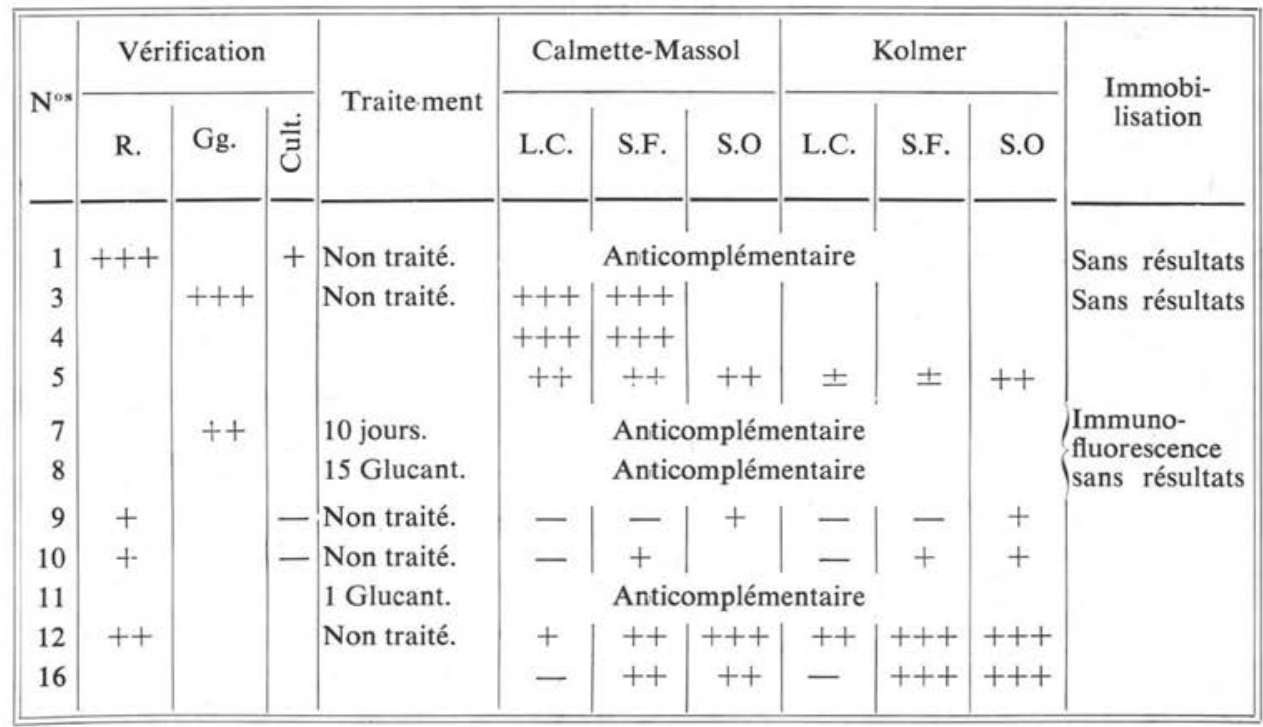


On peut remarquer que, sur les cinq premiers cas humains de kala-azar, une seule fois les réactions se sont montrées positives. Il s'agit d'un malade hospitalisé à MichelLévy et dont l'observation nous a été obligeamment communiquée par le Médecin Lieutenant-Colonel Delahousse. La contamination se serait produite au Tchad, dans une région limitrophe du Soudan anglo-égyptien, à proximité de la région de Dafour qui, d'après Kirk, est une zone importante d'endémie leishmanienne.

On connaît le polymorphisme du kala-azar de l'Est africain, associant souvent des lésions cutanées aux atteintes viscérales. Or, ici, bien que les manifestations cliniques aient été strictement viscérales, la sensibilité de la peau aux antigènes S.O., S.F. et L.C. (les intradermo-réactions étaient toutes positives) et la présence concomitante dans le sérum de sensibilisatrices fixant le complément en présence de tels antigènes semblent confirmer le caractère biologique spécial du parasite de ces régions.

Il en a été de même d'un second malade provenant d'Abeché (Tchad oriental).

Les quatre autres malades n'ont pas réagi avec ces antigènes. Toutefois, il faut noter que l'un d'eux était guéri depuis plus d'un an au moment du prélèvement et que les trois autres étaient en cours de traitement depuis plusieurs jours.

Nous avons vérifié la va'eur de nos antigènes, en pratiquant la réaction de Kolmer chez huit sujets indemnes de parasitose et deux malades ayant un Bordet-Wassermann positif. Seul, l'un des sujets présentant un Bordet-Wassermann dissocié a donné une réaction positive pour S.O.

Chez le chien, les résultats sont beaucoup plus satisfaisants. A'ors qu'avec un sérum de chien normal (22 témoins), on n'obtient pas de fixation de complément, chez onze chiens leishmaniés, les deux réactions pratiquées ont donné sept fois des réponses positives; dans les quatre autres cas, le sérum très anticomp'émentaire rendait la réaction impossib'e.

Chez les hamsters, enfin, les réponses ne sont pas régulières. Sur 45 sérums de hamsters testés, dont six se sont révélés anticomplémentaires, 22 fois nous avons pu faire la réaction de Calmette ; elle s'est presque toujours montrée négative, en accord, dans la moitié des cas seulement, avec la réaction de Kolmer. Cette dernière est positive, 28 fois avec S.O., 21 fois avec S.F., 13 fois avec L.C.

Ici, comme chez le chien, nous avons pu constater que c'est l'antigène S.O. qui réagit le pius souvent et de la façon la p.us intense avec les sérums leishmaniés. L.C. semble présenter des qualités antigéniques inférieures.

\section{Conclusions}

Des différentes préparations antigéniques utilisées au cours des leishmanioses cliniques ou expérimenta!es, il semble que ce soit celles à base de Trypanosoma equiperdum et Strigomonas oncopelti qui aient donné les résultats les plus satisfaisants, tant du point de vue de la sensibilité que celui de la spécificité.

Strigomonas fasciculata et Leptomonas ctenocephali constituent de moins bons antigènes.

Il est assez remarquable que $S$. oncopelti, parasite des Euphorbiacés, soit préciséAnnales de Parasitologie humaine et comparée (Paris), t. 39, 1964, $\mathrm{n}^{\circ} 2$ 
ment un des deux Flagellés qui fournissent le meilleur matériel antigénique pour l'étude des leishmanioses.

Etant donné la facilité avec laquelle on cultive $S$. oncopelti sur bouillon ou sang de lapin et la possibilité de purifier par centrifugation et lavage en eau physiologique ces corps parasitaires, c'est à cette préparation antigénique que nous donnons notre préférence.

Utilisées en intradermo-réaction, les préparations de $T$. equiperdum ou $S$. oncopelti provoquent des réactions al'ergiques notab'es dans le bouton d'Orient, à partir d'un certain stade d'évolution en général, mais quelquefois même au début de l'infestation. Cette allergie cutanée demeure longtemps après la guérison.

Par contre, nous avons enregistré des résultats négatifs au cours des leishmanioses viscérales, sauf chez deux malades qui provenaient de la partie orientale de la République du Tchad, région limitrophe de la République soudanaise.

Cette dernière constatation est fort intéressante, car elle confirme pleinement les observations qu'ont pu faire, sur une vaste éche'le, Kirk et Manson-Bahr au Soudan et au Kenya $(12,13)$.

Il semble donc que le kala-azar est-africain, individualisé par ces deux auteurs, constitue bien une entité clinique et épidémiologique, dont le comportement immunologique est également tout à fait particulier.

Il est difficile de se faire une opinion sur la valeur des intradermo-réactions utilisées chez les chiens ou les hamsters, la structure histologique de leur peau étant, sans doute, peu favorable à ce genre de prospection. Quant aux résultats fournis par les réactions de fixation du complément, ils paraissent plus satisfaisants dans l'étude des leishmanioses expérimentales animales que dans celles des leishmanioses cliniques humaines.

Chez nos malades atteints de bouton d'Orient, ils ont été strictement négatifs, ce qui est normal, étant donné l'absence de lésions viscéra'es. Par contre, nous avons été surpris de ne trouver que deux réactions positives dans les kala-azar, résultats concernant précisément les deux cas d'origine Est africain dont il a été question plus haut.

Dans les kala-azar canins et dans les leishmanioses expérimentales de hamsters infestés avec $L$. donovani infantum, nous avons obtenu une assez forte proportion de résuitats positifs.

Là encore, c'est l'antigène S.O. qui s'est montré le plus sensible.

Pour conclure, il ne semble exister de sensibilisation cutanée chez l'homme que dans le bouton d'Orient et le kala-azar est-africain. Ce qui confirme pleinement les observations de P. E. G. Manson-Bahr (12). Dans ce cas, l'intradermo-réaction peut être effectuée avec toutes sortes de Fiagellés, l'antigène à base de Strigomonas oncopelti donnant des réactions très nettes et étant très facile à préparer constituera le réactif de choix.

Ces antigènes de Flagellés peuvent être également utilisés dans les réactions de fixation du comp'ément, mais i. ne semb'e pas que le kala-azar méditerranéen engendre des anticorps sériques très puissants chez l'enfant ; ces réactions sont présentes chez les chiens infestés naturellement ou chez les hamsters inoculés avec Leishmania donovani infantum. 
Le kala-azar est-africain paraît par contre s'accompagner chez l'homme d'anticorps sériques plus puissants.

\section{Résumé}

Après avoir indiqué les méthodes de préparation d'antigènes à base de Trypanosoma equiperdum, Leptomonas ctenocephali, Strigomonas fasciculata et Strigomonas oncopelti, les auteurs étudient le comportement de ce matériel en intradermo-réactions et en réaction de fixation du complément au cours de diverses leishmanioses cliniques et expérimentales.

Seuls, le bouton d'Orient et le kala-azar est-africain s'accompagnent d'une allergie cutanée, révélée par l'utilisation de ces antigènes. Dans le kala-azar méditerranéen, les intradermo-réactions restent négatives. La réaction de fixation du complément n’a donné des réponses positives que dans les infestations canines ou chez les hamsters inoculés expérimentalement par Leishmania donovani infantum; les sérums provenant d'enfant atteint de kala-azar semblent très pauvres ou dépourvus de sensibilisation fixant le complément en présence de te's antigènes. Il n'en est pas de même dans le kala-azar est-africain.

Parmi les Flagellés étudiés, $T$. equiperdum et $S$. oncopelti constituent les meilleurs antigènes. La facilité de préparation des émulsions de $S$. oncopelti en fait un réactif de choix.

\section{Bibliographie}

1. Sen Gupta (P. C.), 1943. - A complément-fixation Test for Kala-Azar. Indian Med. Gaz., 78, 336-339.

2. - - - 1944. - The value of the Complement-fixation Test in the diagnosis of KalaAzar. Indian Med. Gaz., 79, 465-469.

3. - - - 1945. - Complement-fixation Test with Witebsky, Klingenstein, Kuhn (W.K.K.) or similar antigen: a modified technique. Indian Med. Gaz., 80 396-398.

4. Crosti (A.), Riggio (T.), 1948. - Fenomeni immunitari e valore diagnostico della intradermo reazione con vaccino specifico nella leishmaniosi cutanea. Giorn. Ital. Derm. e Sifilo, 89, 585.

5. Manson-Bahr (P. C. E.), Heish (R. B.), Garnham (P. C. C.), 1959. - Studies in Leishmaniasis East Africa. The Montenegro test in Kala-Azar in Kenya. Trans. Roy. Soc. Trop. Med. a. Hyg., 53, 380-393.

6. Depieds (R.), Collomb (H.), Mathurin (J.), Ranque (J.), 1958. - L'intradermo-réaction à Trypanosoma equiperdum dans le mouton d'Orient. Bulletin Soc. Path. Exot., 51, 501-504.

7. Ranque (J.), Depieds (R.), Collomb (H.), Mathurin (J.), Faure (A.), 1958. - L'intradermo-réaction à antigène $T$. equiperdum dans certaines leishmanioses humaines et animales. Bull. Soc. Path. Exot., 51, 504-506. 
8. Ranque (J.), Depieds (R.), Nicoli (R.-M.), 1960, - Les phénomènes d'immunité dans les leishmanioses. Path. Biol., 8, 1-2, 99.

9. Ranque (J.), in Delahousse (J.), Guyomarc'h (Y.-M.) et Hubert (L.-M.), 1961. - KalaAzar contracté au Tchad par un militaire européen. Méd. Trop., 21, $\mathrm{n}^{\circ}$ 2, 75-79.

10. Dunan (S.), 1961. - Réactions immunologiques dans la Leishmaniose. Thèse Médecine. Marseille.

11. Latysheev (N. I.), Kriukovanb, 1953. - Proc. Reg. exp. Paranto-Med. Zool. Moscow, 8, 211 in Manson-Bahr (P.C.E.).

1961. - Immunity in Kala-Azar. Trans. of the Roy. Soc. Trop. Med. and Hyg., 55, $\mathrm{n}^{\circ}$ 6, nov., 550-55.

12. Manson-Bahr (P.C.E.), 1961. - Immunity in Kala-Azar. Trans. Roy. Soc. Trop. Med. Hyg., 55, 550-55.

13. KIRK (R.), 1939. - Studies in Leishmaniasis in the Anglo-Egyptian Sudan. I. Epidemiology and general considerations. Trans. Roy. Soc. Med. a. Hyg., 32, 533.

14. KIRK (R.), 1944. - Studies in Leishmaniasis in the Anglo-Egyptian Sudan. VI. The evolution of Leishmania infections in man. Trans. Roy. Soc. Med. a. Hyg., 38, 61-70.

15. KIRK (R.), 1950. - Experimental differentiation of Leishmania. Parasit., 40, 58.

Laboratoire de Parasitologie de la Faculté de Médecine, Marseille 